\title{
Dynamic yield stresses of glasses: asymptotic formulae
}

\author{
Oliver Henrich ${ }^{1}$, Fathollah Varnik ${ }^{2}$ and Matthias Fuchs ${ }^{1}$ \\ ${ }^{1}$ Department of Physics, University of Konstanz, Universitätsstrasse 10, D-78457 Konstanz, \\ Germany \\ ${ }^{2}$ Max-Planck-Institut für Eisenforschung, Max-Planck-Strasse 1, D-40237 Düsseldorf, Germany
}

Received 5 October 2005

Published 28 October 2005

Online at stacks.iop.org/JPhysCM/17/S3625

\begin{abstract}
The stationary, shear rate dependent flow curves of yielding glasses are discussed within a mode coupling theory approach. Asymptotic formulae for the shear stress at the transition point are obtained that take the form of generalized Hershel-Bulkeley constitutive equations, and enable one to find dynamical yield stresses by extrapolation.
\end{abstract}

(Some figures in this article are in colour only in the electronic version)

\section{Introduction}

Dense colloidal suspensions exhibit a variety of interesting features when exposed to strong stationary shear flows. The suspensions owe this behaviour to the nonlinear interactions among the particles. At higher packing fractions one can observe a transition from a yielding solid to a shear-thinning fluid, which could be closely related to the glass transition in colloidal liquids. A very promising description of the latter phenomenon in quiescent dispersions has been provided by mode coupling theory (MCT). Yet, sheared systems are driven by external forces and situated quite far off thermal equilibrium. Therefore, the successful MCT approach was recently extended to account for the transient dynamics of suspensions under shear [1]. Starting from the microscopic Smoluchowski equation for interacting Brownian particles under stationary shearing, exact expressions for the shear dependent averages of various quantities have been obtained in the form of Green-Kubo relations. Mode coupling approximations render the equations predictive. The approach assumes a linear flow profile, as has been observed in dense polydisperse systems [2,3], and neglects hydrodynamic interactions. Small shear rates $\dot{\gamma}$ are considered, where shear affects the slow structural rearrangements in the dense dispersion, while it is assumed that solvent-induced effects and symmetry broken states, e.g. shear-banded ones ${ }^{3}$, only occur at higher shear rates.

A state of yielding glass characterized by a finite dynamic yield stress is predicted, which cannot be explained using linear response formalisms. In order to achieve insight into such novel aspects of colloidal systems under shear, simplified MCT models have been formulated

3 Under certain circumstances, flow heterogeneity may occur at low shear rates [4]. 
within the approach that already exhibit universal features of shear thinning, yielding and jamming [5]; first comparisons with experimental data from model systems have supported the approach [6]. In the present contribution we focus on a simple model in order to provide information on how to determine the predicted glassy yield stresses, and compare model results with large scale molecular dynamics simulation data for a binary Lennard-Jones mixture; the latter tests the soundness of our approach.

\section{Theory}

\subsection{Universal aspects and schematic models}

The physics of shear-thinning fluids and yielding solids exhibits qualitative aspects that are solely determined by the underlying transition that separates the two states. Two competing processes have been identified in this respect. On the one hand, with increasing particle interaction or density, the dynamics of the quiescent system slows down tremendously due to the cage effect. Finally, the system ends up in a nonergodic state, in which equilibrium is no longer reached. On the other hand, shearing results in a decorrelation of density fluctuations and leads to a loss of internal stresses. These two different mechanisms introduce two different timescales - one determined by the internal structural dynamics and the other one imposed by the flow. In molecular dynamics simulations of structural glasses, evidence of these specific timescales has been found [7].

Because the transition from shear-thinning fluid to yielding solid occurs in any MCT model that includes the essential bifurcation scenario, it is possible to capture universal aspects by simplified schematic models, if they obey the same stability equations. For instance the extensively studied $F_{12}^{(\dot{\gamma})}$-model considers one normalized correlator, which conforms to a generalized relaxation equation [8]:

$$
\frac{\partial}{\partial t} \Phi(t)+\Gamma\left\{\Phi(t)+\int_{0}^{t} \mathrm{~d} t^{\prime} m\left(t-t^{\prime}\right) \frac{\partial}{\partial t^{\prime}} \Phi\left(t^{\prime}\right)\right\}=0 .
$$

A vanishing memory kernel $m \equiv 0$ would lead to an exponential relaxation of the correlator: $\Phi(t)=\exp (-\Gamma t)$. Memory effects connected with the cage effect cause retardation and a more complicated relaxation behaviour. A simple ansatz suffices to model this:

$$
m(t)=\frac{1}{1+(\dot{\gamma} t)^{2}}\left[v_{1} \Phi(t)+v_{2} \Phi^{2}(t)\right] .
$$

The time dependence of the two vertices $v_{i} /\left(1+(\dot{\gamma} t)^{2}\right) \geqslant 0$ describes the decay of memory caused by shearing. It naturally emerges in the microscopic approach due to the decorrelation of density fluctuations as a consequence of the advection of wavevectors $[1,8,9]$. Increasing vertices $v_{i}$ model the cage effect and lead, in the absence of shear $(\dot{\gamma}=0)$, to glassy (nonergodic) solutions $\Phi(t \rightarrow \infty) \rightarrow f>0$, that arrest at a finite 'glass form factor' $f$ for long times. A typical bifurcation, separating fluid (ergodic, with $\Phi(t \rightarrow \infty)=0$ ) from glassy solutions, lies at $v_{1}^{c}=\sqrt{4 v_{2}^{c}}-v_{2}^{c}$ and $v_{2}^{c}=2$, and we chose the path $v_{1}=v_{1}^{c}+\varepsilon /\left(\sqrt{v_{2}^{c}}-1\right)$ at constant $v_{2}=v_{2}^{c}$ crossing it. The separation parameter $\varepsilon$ in general separates fluid $(\varepsilon<0)$ from glassy $(\varepsilon \geqslant 0)$ states, which with applied shear, however, also decay at long times $\Phi(t \rightarrow \infty, \dot{\gamma} \neq 0)=0$. The transport coefficient of interest, the viscosity $\eta$, and the shear stress will, following Maxwell, be determined by the mean relaxation time of the correlator $\Phi(t)$ :

$$
\sigma=\dot{\gamma} \eta=\dot{\gamma} \int_{0}^{\infty} \mathrm{d} t \Phi(t) .
$$


At very high shear rates, the viscosity is given by the above-mentioned decay rate $\eta_{\infty}=1 / \Gamma$ leading back to the case of exponential relaxation.

\subsection{Yield stresses and scaling laws of yielding glasses}

An interesting consequence of the present approach to steadily sheared glasses is the prediction of a dynamic yield stress; its existence has been shown not only in schematic models but also in the full microscopic framework [1]. The ideal glass state of classical MCT [10] is shear melted with even an infinitesimal shear rate $\dot{\gamma}$ provided one waits long enough so that the system can reach the stationary state after the decay of the transients. Because the transients at finite $\dot{\gamma}$ possess $\tau_{\dot{\gamma}}=c_{s} /|\dot{\gamma}|$ as the longest relaxation time (with constant $c_{s}$ that takes the value $c=0.594$ for the $F_{12}^{(\dot{\gamma})}$-model), reaching the stationary state should always be possible by applying finite shear rates during the preparation of the system. Starting with large shear rates to melt the solid state or to prevent arrest during a quench into glassy states, and then reducing the shear rate, allows one to approach the limit of vanishing shear rate where a yielding of the glassy state is predicted regardless of how small $\dot{\gamma}$ becomes. The stress does not fall below a finite yield stress value because the decay of the transients requires driving by shear.

These aspects can be analysed in detail close to the glass transition, namely for $|\varepsilon| \ll$ $\varepsilon_{\dot{\gamma}}=\left|\dot{\gamma} t_{0}\right|^{\tilde{m}} \ll 1$, where-as shown in the appendix-the $F_{12}^{(\dot{\gamma})}$-model is solved at long times by the expansion

$$
\Phi(t, \dot{\gamma}, \varepsilon)=\Phi^{+}(\tilde{t})+\left|\dot{\gamma} t_{0}\right|^{m} \Phi^{+(1)}(\tilde{t})+\left|\dot{\gamma} t_{0}\right|^{2 m} \Phi^{+(2)}(\tilde{t})+\cdots,
$$

where $\tilde{t}=t / \tau_{\dot{\gamma}}$, and the $\Phi^{+}$and $\Phi^{+(i)}$ are $\varepsilon$ - and $\dot{\gamma}$-independent functions introduced in equation (A.1) in the appendix. The parameter $t_{0}$ is a matching time, set by the crossover to the initial decay connected with $\Gamma$; in the model $t_{0} \Gamma=0.43$ is found. The exponents are given by $\tilde{m}=\frac{2 a}{1+a}, m^{\prime}=\frac{2 \lambda-1}{2 \lambda}$ and $m=\tilde{m} m^{\prime}$, with the familiar MCT exponents [10], that take the values $\lambda=0.707$ and $a=0.324$ at the chosen transition, yielding $m=0.143$ and $\tilde{m}=0.489$. Clearly, the scaling functions, which describe the final relaxation of the transients, exhibit the claimed shear-induced relaxation time $\tau_{\dot{\gamma}}$. The power-law appearance of $\dot{\gamma}$ in the coefficients of the expansion arises from the nonlinear stability equation describing the shear melting of the glassy structure [8]. Inserting this expansion into equation (3) gives the asymptotic expansion of the stress curves close to the glass transition or for not too small shear rates. It takes the form of a generalized Hershel-Bulkeley constitutive equation:

$$
\sigma\left(\dot{\gamma}, \varepsilon \ll \varepsilon_{\dot{\gamma}}\right)=\sigma_{c}^{+}\left(1+\left|\dot{\gamma} / \dot{\gamma}_{*}\right|^{m}+c_{2}\left|\dot{\gamma} / \dot{\gamma}_{*}\right|^{2 m}+c_{3}\left|\dot{\gamma} / \dot{\gamma}_{*}\right|^{3 m}+\cdots\right),
$$

where $\dot{\gamma}_{*}$ is an upper limit to the shear rate where this expansion holds because of the requirement $\left|\dot{\gamma} / \dot{\gamma}_{*}\right| \propto\left|\dot{\gamma} t_{0}\right| \ll 1$; the $F_{12}^{(\dot{\gamma})}$-model gives $\dot{\gamma}_{*}=10^{-2.41} \Gamma$, and $\sigma_{c}^{+}=0.10$, $c_{2}=0.896$, and $c_{3}=0.950$. Figure 1 shows numerically obtained stress versus shear rate curves in yielding glassy states, $\varepsilon \geqslant 0$, and the (fitted) expansion from equation (5). (The fit gives the values for the parameters $c_{i}$ reported above.) It describes well the critical shear curve (at $\varepsilon=0$ ), and curves in the glass for not too small shear rates, $\dot{\gamma} \gg \dot{\gamma}_{*}|\varepsilon|^{1 / m}$.

Deeper in the glass or for smaller $\dot{\gamma}$ than estimated above, the yield stress exhibits a $\varepsilon$-dependent plateau that rises quickly and is independent of $\dot{\gamma}[8]$ :

$$
\sigma^{+}(\varepsilon)=\sigma\left(\dot{\gamma}, \varepsilon \gg \varepsilon_{\dot{\gamma}}\right)=\sigma_{c}^{+}+\sigma^{\prime} \sqrt{\varepsilon}+\cdots .
$$

Numerical results from the $F_{12}^{(\dot{\gamma})}$-model shown in figure 1 clearly exhibit this law for small enough $\varepsilon$. 


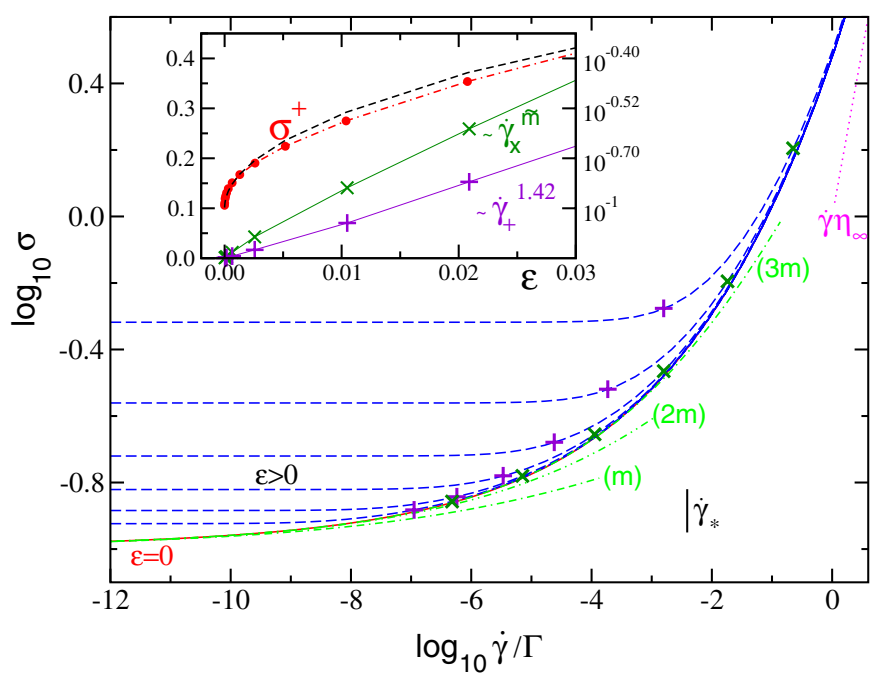

Figure 1. Steady state stress versus shear rate curves, $\sigma(\dot{\gamma})$, for glassy states $(\varepsilon \geqslant 0)$ in the $F_{12}^{(\dot{\gamma})}$-model. The separation parameters are $\varepsilon=0$ (solid line; hidden by other lines), and $\log _{10} \varepsilon=-4.39,-3.79,-3.19,-2.59,-1.98$, and -1.38 from bottom to top. The $\varepsilon$-independent asymptotes from equation (5) are shown as chain curves labelled with the highest power included. The high shear rate behaviour is also indicated. Plus signs mark where the stresses have increased by $10 \%$ above the yield value, $\sigma\left(\dot{\gamma}_{+}, \varepsilon\right)=1.1 \sigma^{+}(\varepsilon)$. Small times signs mark where the shear rates of glass curves differ horizontally by $20 \%$ from the curve at $\varepsilon=0, \sigma\left(\dot{\gamma}_{x}, \varepsilon\right)=\sigma(1.2 \dot{\gamma}, \varepsilon=0)$. The inset shows the dynamic yield stresses versus the separation parameter, $\sigma^{+}(\varepsilon)$, and the square root asymptote (dashed line) from equation (6). Also shown are the crossover shear rates from the estimates in the main panel; they show power-law behaviour, $\dot{\gamma}_{+} \sim \varepsilon^{0.70}$ and $\dot{\gamma}_{x} \sim \varepsilon^{1 / \tilde{m}}$.

\section{Simulation data and analysis with the schematic model}

In large scale molecular dynamics simulations a 80:20 binary mixture of Lennard-Jones particles at constant density was used. This model has well known equilibrium properties and many aspects that can be understood consistently within MCT [11]. To account for shearing, it was used together with Lees-Edwards boundary conditions and the SLLOD equations of motion to develop a linear velocity profile. (Note that in the simulation, solvent effects are obviously lacking, and the simulated flow curves thus provide support for the notion that shear thinning can arise from shear-induced speeding up of the structural relaxation.)

Figure 2 shows the stress-shear rate dependence as flow curves, for temperatures ranging from supercooled states to the glassy regime. The solid lines are fits to the simulation data with the $F_{12}^{(\dot{\gamma})}$-model, which reproduce the transition from a shear-thinning fluid to a yielding solid quite well. Coming from high shear rates, the flow curves of the supercooled state pass to the linear response regime in the lower left corner, indicated by a dasheddotted line with slope 1 . On approaching the transition point, the linear response regime shifts to lower and lower shear rates. Beyond the fluid domain, the existence of a dynamic yield stress $\sigma^{+}=\lim _{\dot{\gamma} \rightarrow 0} \sigma>0$ is supported by the simulation results, which sustain the discontinuous change in dynamic yield stress and generate a stress plateau over three decades in shear rate. Best $F_{12}^{(\dot{\gamma})}$-model fits are obtained for a $T_{\mathrm{c}}=0.4$ suggesting a slightly lower transition temperature $[5,12]$ as determined from the simulations of the quiescent system, where $T_{\mathrm{c}}=0.435$ was found [11]. 


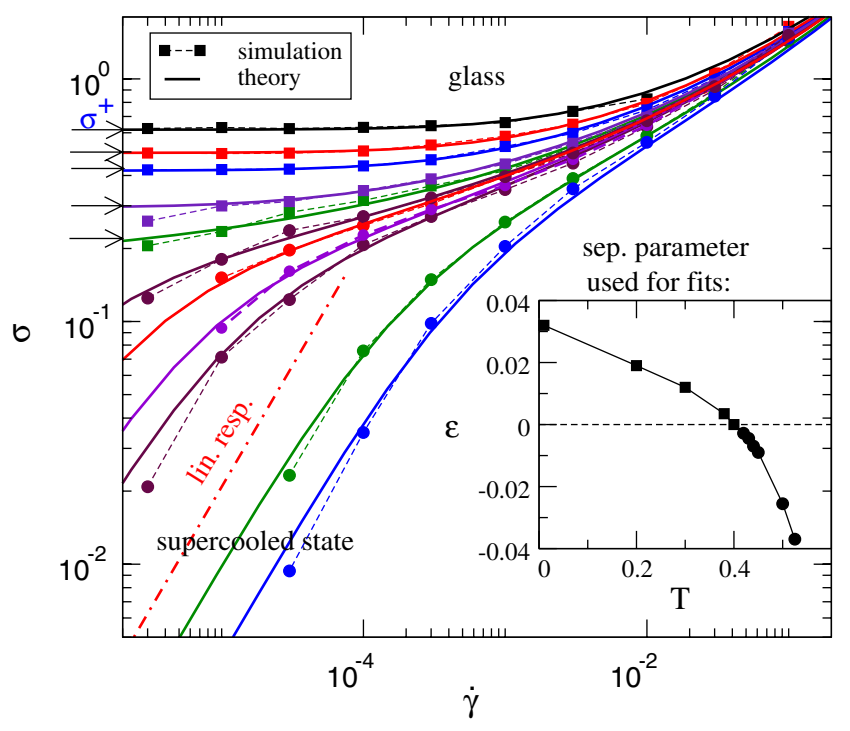

Figure 2. Flow curves $\sigma(\dot{\gamma})$ reaching from the supercooled to the glassy state of a simulated binary LJ mixture. The data points correspond to the temperatures $T=0.525,0.5,0.45,0.44,0.43,0.42$, $0.4,0.38,0.3,0.2$, and 0.01 in LJ units (from bottom to top). $F_{12}^{(\dot{\gamma})}$-model curves fitted by eye are included as lines. The inset shows the relation between the fitted separation parameters and temperature. Units are converted using $\sigma=1.5 \sigma_{\text {theo }}$ and $\dot{\gamma}=1.3 \dot{\gamma}_{\text {theo }} \Gamma$; from [13]. The arrows mark the values of the extrapolated dynamic yield stresses $\sigma^{+}(\varepsilon)$.

\section{Acknowledgments}

$\mathrm{OH}$ was supported by the Deutsche Forschungsgemeinschaft (DFG) (in the International Graduate College 'Soft Matter'), FV by the DFG under grant VA 205/1-1, and MF by the DFG Transregio-SFB TR6.

\section{Appendix}

In this appendix the derivation of equation (4) within the $F_{12}^{(\dot{\gamma})}$-model is sketched; for more details see [8, 14]. Close to the transition, namely for $\varepsilon \rightarrow 0$, and for small shear rates, $|\dot{\gamma}| \rightarrow 0$, the correlator develops a plateau for intermediate times, $\Phi(t)=f_{c}+\left(1-f_{c}\right)^{2} \mathcal{G}(t)+\cdots$, where the slowly varying scaling function $\mathcal{G}$ obeys the (universal—within the approach of [1]) stability equation that describes the yielding of a glass. Close to the transition, $|\varepsilon| \ll \varepsilon_{\dot{\gamma}} \ll 1$, and for times $t$ in between $t_{\dot{\gamma}}=t_{0} \varepsilon_{\dot{\gamma}}^{-1 / 2 a} \ll t \ll \tau_{\dot{\gamma}}$, the function $\mathcal{G}$ possesses the expansion [8]

$$
\begin{array}{r}
\mathcal{G}=\sqrt{\varepsilon_{\dot{\gamma}}} \breve{\mathcal{G}}\left(t / t_{\dot{\gamma}}\right)=-\frac{\sqrt{\varepsilon_{\dot{\gamma}}}}{c_{s}} \frac{t}{t_{\dot{\gamma}}}\left[1-\hat{\alpha}_{1}\left(\frac{t}{t_{\dot{\gamma}} c_{s}}\right)^{-2 m^{\prime}}-\hat{\alpha}_{2}\left(\frac{t}{t_{\dot{\gamma}} c_{s}}\right)^{-4 m^{\prime}}+\cdots\right] \\
=-\frac{t}{\tau_{\dot{\gamma}}}\left[1-\left|\dot{\gamma} t_{0}\right|^{m} \hat{\alpha}_{1}\left(\frac{t}{\tau_{\dot{\gamma}}}\right)^{-2 m^{\prime}}-\left|\dot{\gamma} t_{0}\right|^{2 m} \hat{\alpha}_{2}\left(\frac{t}{\tau_{\dot{\gamma}}}\right)^{-4 m^{\prime}}+\cdots\right] .
\end{array}
$$

This expansion can be considered as the long time asymptote of the dynamics around the plateau $f_{c}$, or as the short time asymptote of the final relaxation process leading down from $f_{c}$ to zero. In the latter context, considering the higher order corrections $\Phi-f_{c} \propto \breve{\mathcal{G}}+\varepsilon_{\dot{\gamma}} \breve{\mathcal{G}}^{(2)}+\cdots$, one recognizes that each order $\breve{\mathcal{G}}^{(i)}$ possesses a long time expansion like equation (A.1), 
containing the powers $\left|\dot{\gamma} t_{0}\right|^{m}$. Resumming the series leads to equation (4), with known short time expansions of the functions:

$\Phi^{+}(\tilde{t} \rightarrow 0) \rightarrow f_{c}-\left(1-f_{c}\right)^{2} \tilde{t}, \quad \Phi^{+(i)}(\tilde{t} \rightarrow 0) \rightarrow\left(1-f_{c}\right)^{2} \hat{\alpha}_{i} \tilde{t}^{1-2 i m^{\prime}}$,

which shows that the expansion coefficients $c_{i}$ in equation (5) can be calculated from convergent integrals as long as $i<1 / m^{\prime}$, which gives $i \leqslant 3$ for the $F_{12}^{(\dot{\gamma})}$-model.

\section{References}

[1] Fuchs M and Cates M E 2002 Phys. Rev. Lett. 89248304 Fuchs M and Cates M E 2005 J. Phys.: Condens. Matter 171681

[2] Derks D, Wisman H, van Blaaderen A and Imhof A 2004 J. Phys.: Condens. Matter 163917

[3] Petekidis G, Moussaid A and Pusey P 2002 Phys. Rev. E 66051402

[4] Varnik F, Bocquet L, Barrat J-L and Berthier L 2003 Phys. Rev. Lett. 90095702

[5] Cates M E, Holmes C, Fuchs M and Henrich O 2004 Unifying Concepts in Granular Media and Glasses ed A Coniglio et al (Amsterdam: Elsevier) p 203 (Preprint cond-mat/0310579)

[6] Fuchs M and Ballauff M 2004 J. Chem. Phys. 122094707

[7] Berthier L and Barrat J L 2002 J. Chem. Phys. 1166228

[8] Fuchs M and Cates M E 2003 Faraday Discuss. 123267

[9] Miyazaki K and Reichman D R 2002 Phys. Rev. E 66 050501(R)

[10] Götze W 1991 Liquids, Freezing and Glass Transition ed J-P Hansen, D Levesque and J Zinn-Justin (Amsterdam: North-Holland) p 287

[11] Kob W and Andersen H C 1995 Phys. Rev. E 514626

Kob W and Andersen H C 1995 Phys. Rev. E 524134

[12] Flenner E and Szamel G 2005 Phys. Rev. E 72011205

[13] Varnik V 2005 Nonlinear rheology and dynamic yielding, in preparation

[14] Henrich O and Fuchs M 2005 Stability analysis of sheared glasses, in preparation 